\title{
Finite Element Approximation to a Finite-Size Modified Poisson-Boltzmann Equation
}

\author{
Jehanzeb Hameed Chaudhry • Stephen D. Bond • \\ Luke N. Olson
}

Received: 22 February 2010 / Revised: 31 October 2010 / Accepted: 18 November 2010

(C) Springer Science+Business Media, LLC 2010

\begin{abstract}
The inclusion of steric effects is important when determining the electrostatic potential near a solute surface. We consider a modified form of the Poisson-Boltzmann equation, often called the Poisson-Bikerman equation, in order to model these effects. The modifications lead to bounded ionic concentration profiles and are consistent with the PoissonBoltzmann equation in the limit of zero-size ions. Moreover, the modified equation fits well into existing finite element frameworks for the Poisson-Boltzmann equation. In this paper, we advocate a wider use of the modified equation and establish well-posedness of the weak problem along with convergence of an associated finite element formulation. We also examine several practical considerations such as conditioning of the linearized form of the nonlinear modified Poisson-Boltzmann equation, implications in numerical evaluation of the modified form, and utility of the modified equation in the context of the classical PoissonBoltzmann equation.
\end{abstract}

Keywords Finite elements · Poisson-Boltzmann · Poisson-Bikerman

\section{Introduction}

Electrostatic interactions play a critical role in determining macroscopic properties of biomolecular systems, such as solvation free energy and binding affinities [1-3]. The Poisson-

Research of J.H. Chaudhry was supported by the University of Illinois Computational Science and Engineering Fellowship Program. Research of S.D. Bond was supported in part by NSF-CCF 08-30578. Research of L.N. Olson was supported in part by NSF-DMS 07-46676.

J.H. Chaudhry $(\varangle) \cdot$ L.N. Olson

Department of Computer Science, University of Illinois at Urbana-Champaign, Urbana, IL 61801, USA e-mail: jhameed2@illinois.edu

L.N. Olson

e-mail: lukeo@illinois.edu

S.D. Bond

Applied Mathematics and Applications Group, Sandia National Laboratories, Albuquerque, NM 87185, USA

e-mail: sdbond@sandia.gov

Published online: 01 December 2010 
Boltzmann equation (PBE), introduced decades ago [4, 5], has proved successful in approximating electrostatic interactions for moderate surface charge densities. The PBE adopts a continuum mean-field description of a solvent, assuming point-like ions in thermodynamic equilibrium, and neglecting statistical correlations [6]. However, the assumption of zero-size ions in this description leads to unphysical concentrations near the surface under moderate charge densities, even if the bulk solution is dilute [7].

One approach to modeling the effects of finite-size ions is to consider a lattice gas model for the free ions [7], which leads to the modified PBE (MPBE). Also termed the PoissonBikerman equation [8,9], the MPBE has the advantage of bounded concentrations of ions near the molecular surface. As the size of the ions tend toward zero, the modified equation converges to the PBE. The value of incorporating steric effects into the equation is highlighted through several recent applications that have adopted the MPBE [7, 8, 10, 11].

In addition to the MPBE, there have been other attempts to modify the PoissonBoltzmann theory in an effort to account for steric effects. For example, through a series of modified equations [12], a new model has been derived [13], which takes into account volume-exclusion effects of free ions. In another model based on density functional theory [14], in addition to the ion-exclusion effects, solvent exclusion effects are approximated by modeling solvent molecules as neutral, hard spheres. While effective, these alternate approaches involve non-trivial equations, and considerable computational effort is required to solve them. One advantage of the MPBE proposed by [7], and considered here, is in the simplicity and ease with which it can be incorporated with existing PBE numerical implementations.

The finite element method has been widely used for solving the PBE [15-23]. In this paper we analyze the MPBE. We start by analytically subtracting the singularities in the potential through a common regularization procedure $[15,24]$ and follow by proving the existence and uniqueness of weak solutions to the regularized MPBE. We then examine the finite-element formulation for this equation and show that the corresponding approximation converges as the mesh is refined. As a practical consideration, we also examine the conditioning of linear systems which arise during discretization. Finally, we discuss a method for solving the unmodified PBE by first solving the modified equation to improve the initial guess. We argue that this approach is vital for fast convergence of Newton-like methods.

The unmodified PBE has been analyzed by Chen, Holst, and $\mathrm{Xu}$ [15]. Although our analysis of the modified PBE uses a similar approach, there are important differences. In particular, the nonlinearity in the modified equation is bounded, which simplifies the theory. When applicable, we use prior results for the unmodified PBE to contribute to our theory and discussion.

A slightly different form of the modified Poisson-Boltzmann equation has been analyzed by $\mathrm{Li}$ [25]. The focus is on deriving the equation from a free energy function, from properties of equilibrium concentrations, and by rigorously proving equivalence of different forms of the equation. In this paper, we approach existence and uniqueness of solution differently, using results from convex optimization. Moreover, our results focus on discrete solutions to the problem.

The remainder of the paper is organized as follows. In Sect. 2 we describe the MPBE, its domain, and different transformations to make it amenable for numerical computations. In Sect. 3 we prove the existence of a unique weak solution to the regularized problem. We give a finite-element formulation for regularized MPBE in Sect. 4 and show optimality and convergence of the approximations. Finally, in Sect. 5, we discuss a relationship between the PBE and the MPBE as ion size is decreased in the MPBE. 


\section{The Modified Poisson Boltzmann Equation}

Biomolecular systems are frequently modeled through classical molecular dynamics, where the dynamics of the atoms in the system are resolved, and thermodynamic properties are estimated by averaging in time [26]. Due to the large number of solvent molecules, and the small timesteps required in the simulation, explicit methods based on molecular dynamics are extremely expensive $[27,28]$. A more tractable approach is to represent the solvent implicitly as a dielectric continuum, which results in the Poisson-Boltzmann model. Let $\phi(x)$ represent the unknown electrostatic potential, $\epsilon(x)$ the dielectric coefficient, and $\bar{\kappa}(x)$ the modified Debye-Hückel parameter. Defining constant $\beta=1 /\left(k_{B} T\right), k_{B}$ as the Boltzmann constant, $e_{c}$ as the charge of an electron, and $T$ as the temperature, the Poisson-Boltzmann equation for 1:1 solution (one positive and one negative ion per salt molecule, e.g. $\mathrm{NaCl}$ ) is,

$$
\begin{aligned}
& -\nabla \cdot(\epsilon(x) \nabla \phi(x))+\frac{1}{\beta e_{c}} \bar{\kappa}^{2}(x) \sinh \left(\beta e_{c} \phi(x)\right)=4 \pi \sum_{i=1}^{N_{m}} Q_{i} \delta\left(x-x_{i}\right) \text { in } \mathbb{R}^{3}, \\
& \phi(\infty)=0 .
\end{aligned}
$$

Here, the solute contains $N_{m}$ fixed points with charges $Q_{i}$ at positions $x_{i}$, and $\delta$ represents the Dirac delta distribution.

The domain for the problem is $\mathbb{R}^{3}$ which is subdivided into a molecular region $\Omega_{m}$, a solvent region $\Omega_{s}^{\infty}$, and an interface between the two denoted by $\Gamma$. The solute is surrounded by solvent, which is represented as a continuum over the subdomain $\Omega_{s}^{\infty}=\mathbb{R}^{3} \backslash \bar{\Omega}_{m}$. The subdomains for a typical biomolecular solute are shown on the left in Fig. 1.

Let $\epsilon_{m}$ and $\epsilon_{s}$ be positive constants, and $N_{A}$ represent Avogadro's number. The dielectric coefficient, $\epsilon(x)$, and modified Debye-Hückel parameter, $\bar{\kappa}^{2}(x)$, are piecewise constant functions given by

$$
\epsilon(x)=\left\{\begin{array}{ll}
\epsilon_{m}, & x \in \Omega_{m}, \\
\epsilon_{s}, & x \in \Omega_{s}^{\infty},
\end{array} \quad \text { and } \quad \bar{\kappa}^{2}(x)= \begin{cases}0, & x \in \Omega_{m}, \\
\bar{\kappa}_{s}^{2}=\epsilon_{s} \frac{8 \pi N_{A} e_{c}^{2}}{1000 k_{B} T} c_{b}, & x \in \Omega_{s}^{\infty} .\end{cases}\right.
$$

In the MPBE, the free ions occupy cells in a three-dimensional lattice of size $a$, as shown on the right in Fig. 1. Let $a$ be the diameter of an ion and $c_{b}$ be the bulk concentration or ionic strength (which depends on the particular solvent being modeled). Defining $v=2 a^{3} c_{b}$, the modified PBE for a 1:1 solution, obtained from [7] (with a straightforward extension to
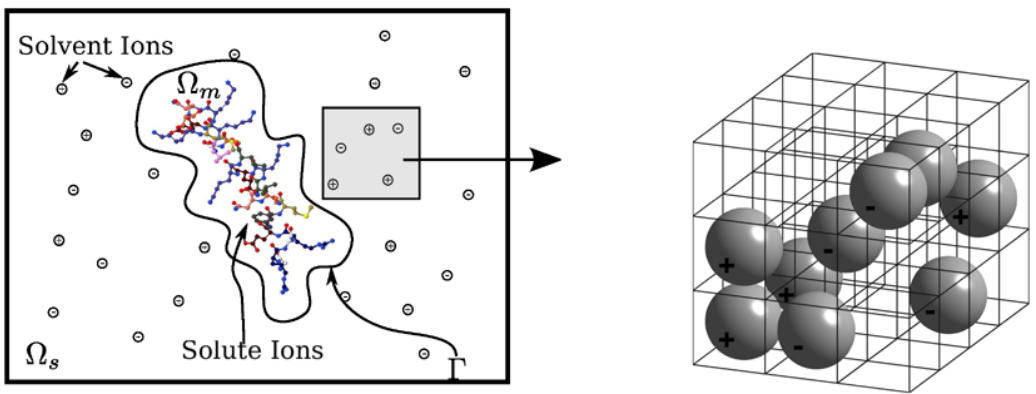

Fig. 1 Subdomains for the Poisson-Boltzmann equation 


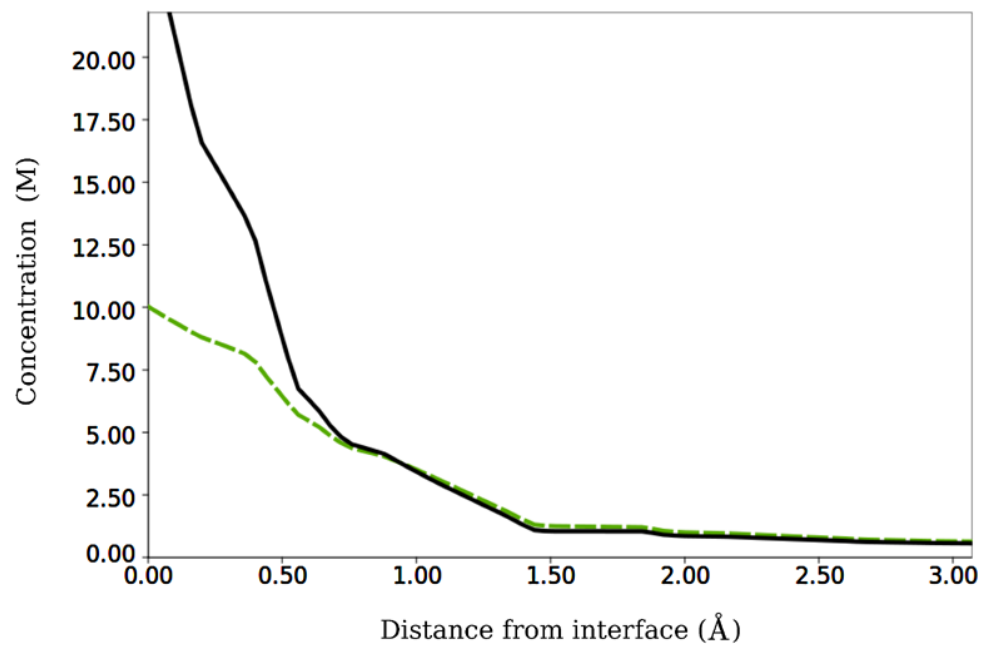

Fig. 2 Concentration profile near the Born ion interface

account for the molecular region as is done for unmodified PBE in such cases) is,

$$
\begin{aligned}
& -\nabla \cdot(\epsilon(x) \nabla \phi(x))+\frac{1}{\beta e_{c}} \bar{\kappa}^{2}(x) \frac{\sinh \left(\beta e_{c} \phi(x)\right)}{1-v+v \cosh \left(\beta e_{c} \phi(x)\right)} \\
& \quad=4 \pi \sum_{i=1}^{N_{m}} Q_{i} \delta\left(x-x_{i}\right), \quad \text { in } \mathbb{R}^{3}, \\
& \phi(\infty)=0 .
\end{aligned}
$$

For the remainder of this paper, we assume that $0<v<1$, unless stated otherwise. Notice that as $a \rightarrow 0$ we recover the PBE.

The assumption of zero-size ions in the unmodified PBE leads to unphysical concentrations near the surface. This is illustrated in Fig. 2 for the Born ion (described in Sect. 4.2), which shows counter-ion concentrations calculated using our finite-element formulation with a free ion size of $5 \AA$ and bulk concentration of $0.1 \mathrm{M}$. The PBE results in high concentrations of counter-ions near the surface of the molecule, whereas the MPBE yields a realistically bounded profile.

\subsection{Truncation}

For computation, the unbounded solvent domain, $\Omega_{s}^{\infty}$, is truncated to a bounded domain, $\Omega_{s}$, and the resulting problem domain, $\Omega=\Omega_{s} \cup \Gamma \cup \Omega_{m}$, has a convex and Lipschitzcontinuous boundary $\partial \Omega$. Dirichlet boundary conditions are imposed to capture the asymptotic behavior of the solution on an unbounded domain. Together with a change of variables, $\tilde{u}(x)=\beta e_{c} \phi(x)$, this results in a dimensionless modified Poisson-Boltzmann equation on $\Omega$ :

$$
\begin{aligned}
& -\nabla \cdot(\epsilon(x) \nabla \tilde{u}(x))+\bar{\kappa}^{2}(x) \frac{\sinh (\tilde{u}(x))}{1-v+v \cosh (\tilde{u}(x))} \\
& \quad=4 \pi \beta e_{c} \sum_{i=1}^{N_{m}} Q_{i} \delta\left(x-x_{i}\right) \quad \text { in } \Omega \\
& \tilde{u}(x)=g(x) \text { on } \partial \Omega .
\end{aligned}
$$


The boundary conditions are prescribed using a linear combination of Helmholtz Green's functions,

$$
g(x)=\beta e_{c} \sum_{i=1}^{m} \frac{Q_{i}}{\epsilon_{s}\left|x-x_{i}\right|} \exp \left(\frac{-\bar{\kappa}_{s}\left|x-x_{i}\right|}{\sqrt{\epsilon_{s}}}\right),
$$

which captures the asymptotic behavior of the solution. This boundary condition is the same as boundary conditions commonly used for the PBE [15] and can be derived from the asymptotic properties of (2).

Similar to the unmodified case, the solution to the modified PBE must satisfy two conditions at the interface $\Gamma$,

$$
\llbracket \tilde{u}(x) \rrbracket_{\Gamma}=0 \quad \text { and } \quad \llbracket \epsilon(x) \frac{\partial \tilde{u}(x)}{\partial \mathbf{n}} \rrbracket_{\Gamma}=0, \quad x \in \Gamma,
$$

where $\mathbf{n}$ is the unit normal at the interface and the jump at the interface is defined as

$$
\llbracket v(x) \rrbracket_{\Gamma}=\lim _{\alpha \rightarrow 0^{+}} v(x+\alpha \mathbf{n})-v(x-\alpha \mathbf{n}) .
$$

\subsection{Regularization}

Equation (2) resembles a standard second order elliptic partial differential equation, but the right hand side contains delta distributions which are not in $H^{-1}(\Omega)-$ i.e., the dual space of $H_{0}^{1}(\Omega)$ (see [15]). This precludes seeking a solution in $H^{1}(\Omega)$, the typical Sobolev space for second order equations. The lack of smoothness of the solution $\tilde{u}$ also hinders the design of a convergent finite element method for the MPBE. Following [24], we overcome this issue by decomposing $\tilde{u}$ into

$$
\tilde{u}=u+u_{c}
$$

where $u$ is an unknown smooth function and $u_{c}$ is a known singular function which absorbs the singularities in $\tilde{u}$. The Coulomb function, $u_{c}$, satisfies the Poisson equation

$$
-\epsilon_{m} \nabla \cdot \nabla u_{c}(x)=4 \pi e_{c} \beta \sum_{i=1}^{m} Q_{i} \delta\left(x-x_{i}\right) .
$$

Combining (6) with (2), we obtain the regularized MPBE or RMPBE:

$$
\begin{aligned}
& -\nabla \cdot(\epsilon(x) \nabla u(x))+\bar{\kappa}^{2}(x) \frac{\sinh \left(u(x)+u_{c}(x)\right)}{1-v+v \cosh \left(u(x)+u_{c}(x)\right)} \\
& \quad=\nabla \cdot\left(\left(\epsilon(x)-\epsilon_{m}\right) \nabla u_{c}(x)\right) \quad \text { in } \Omega, \\
& u(x)=g(x)-u_{c}(x) \quad \text { on } \partial \Omega .
\end{aligned}
$$

\section{Existence and Uniqueness}

In this section we establish the existence and uniqueness of the weak solution to the RMPBE. Although our approach is similar to the approach used by Chen, Holst, and $\mathrm{Xu}$ [15] to analyze the unmodified RPBE, the assumptions differ and we are able to make several simplifications to make the theory more accessible. In particular, we do not need an $L^{\infty}$ bound since the nonlinearity in the modified equation is bounded. 
We denote the $L^{2}(\Omega)$ inner product by $(.,$.$) and the duality pairing between functions$ from $H_{0}^{1}(\Omega)$ and $H^{-1}(\Omega)$ by $\langle.,$.$\rangle . Define$

$$
M:=\left\{v \in H^{1}(\Omega) \mid v=g-u_{c} \text { on } \partial \Omega\right\}
$$

and $V=H_{0}^{1}(\Omega)$. The weak problem for (8) becomes: Find $u \in M$ such that

$$
A(u, v)+(N(u), v)+\left\langle f_{c}, v\right\rangle=0, \quad \forall v \in V,
$$

where

$$
\begin{aligned}
A(u, v) & =(\epsilon \nabla u, \nabla v), \\
(N(u), v) & =\left(\bar{\kappa}^{2} \frac{\sinh \left(u+u_{c}\right)}{1-v+v \cosh \left(u+u_{c}\right)}, v\right), \quad \text { and } \\
\left\langle f_{c}, v\right\rangle & =\int_{\Omega}\left(\epsilon-\epsilon_{m}\right) \nabla u_{c} \cdot \nabla v d \mathbf{x} .
\end{aligned}
$$

For (11) to be well defined, we need $N(u) \in L^{2}(\Omega)$. This is true since $N(u) \in L^{\infty}(\Omega)$, which follows from the bound

$$
\frac{\sinh \left(u+u_{c}\right)}{1-v+v \cosh \left(u+u_{c}\right)} \leq \frac{1}{v} \quad \text { in } \Omega
$$

We seek an energy functional on $\mathrm{M}$ with a minimum that satisfies (11). Define $E$ as

$$
E(w)=\int_{\Omega} \frac{\epsilon}{2} \nabla w \cdot \nabla w+\frac{\bar{\kappa}^{2}}{v} \ln \left(1-v+v \cosh \left(w+u_{c}\right)\right) d \mathbf{x}+\left\langle f_{c}, w\right\rangle .
$$

The motivation for choosing this energy is given by the following lemma, which shows that the solution to the RMPBE is the minimizer of this energy.

Lemma 3.1 If $u$ is the solution of the optimization problem, i.e.

$$
E(u)=\inf _{w \in M} E(w),
$$

then $u$ is the solution of (11).

Proof Similar to the proof of Lemma (4.1) in [15], we consider the function $F: \mathbb{R} \rightarrow \mathbb{R}$ given by

$$
F(\tau)=E(u+\tau v),
$$

for any $v \in H_{0}^{1}(\Omega)$. Taking the derivative of this function at its critical point $(\tau=0)$ proves the assertion.

With a suitable energy function defined, the following theorem guarantees the existence and uniqueness of the solution to the minimization problem (13).

Theorem 3.2 (Existence and uniqueness) There exists a unique $u \in M \subset H^{1}(\Omega)$ such that

$$
E(u)=\inf _{w \in M} E(w) .
$$


To prove this theorem we make use of results from variational analysis. The requisite definitions and theorems that we use are now given. Let $f: V \rightarrow \mathbb{R}$ be a functional on a separable reflexive Banach space $V$ with norm $\|\cdot\|$. We denote convergence in norm by $\rightarrow$ and weak convergence by $\rightarrow$. Additionally, recall that a functional $f$ is lower semicontinuous at $v \in V$ if $f(v) \leq \liminf _{n \rightarrow \infty} f\left(v_{n}\right)$ for any sequence $\left\{v_{n}\right\}$ such that $v_{n} \rightarrow v$ and is weakly lower semi-continuous at $v \in V$ if $f(v) \leq \liminf _{n \rightarrow \infty} f\left(v_{n}\right)$ for any sequence $\left\{v_{n}\right\}$ such that $v_{n} \rightarrow v$. We also make use of coercive and proper functions. A function $f: V \rightarrow \mathbb{R} \cup\{\infty\}$ is said to be coercive if $\lim _{\|x\| \rightarrow \infty} f(x)=\infty$, and is proper if $f \neq \infty$. With these standard definitions, we are ready to prove Theorem 3.2.

Proof of Theorem 3.2 To prove existence of minimizer, we need to verify that $M$ is a convex set, and $E$ is a convex, coercive and semi lower-continuous functional on $M$. To show convexity of $M$, we consider $w=t u+(1-t) v$ for any $u, v \in M$ and a scalar $t$ such that $0<t<1$. Since $M \subset H^{1}(\Omega)$, we have $w \in H^{1}(\Omega)$ as $H^{1}(\Omega)$ is a linear space. On the boundary, $\partial \Omega$, we have

$$
w=t u+(1-t) v=t\left(g-u_{c}\right)+(1-t)\left(g-u_{c}\right)=g-u_{c} \quad \text { on } \partial \Omega,
$$

and hence we conclude that $w \in M$. This shows that the set $M$ is convex.

From the proof of Lemma 3.1, it follows that $E(w)$ is Gâteaux differentiable with

$$
D E(u)[v]=A(u, v)+(N(u), v)+\left\langle f_{c}, v\right\rangle .
$$

Thus from Theorem 7.2.3 in [29], $E$ is weakly lower semi-continuous. Since $E$ is a proper function, i.e. $E(0) \neq \infty$, then Theorem 3.3.3 in [30] immediately implies that it is lower semi-continuous.

The convexity of $E$ follows from the convexity of $x^{2}$ and the convexity of $r(t)=\ln (1-$ $v+v \cosh (t))$ as a function from $\mathbb{R}$ to $\mathbb{R}$. The convexity of latter function is seen from

$$
r^{\prime \prime}(t)=v \frac{v+(1-v) \cosh (t)}{(1-v+v \cosh (t))^{2}}>0,
$$

since $0<v<1$. Thus $E$ is strictly convex.

To show coercivity of $E$, we have by a generalization of the arithmetic-geometric mean inequality, for any $\delta>0$

$$
\left\langle f_{c}, v\right\rangle \leq \epsilon_{s}\left\|\nabla u_{c}\right\|_{\Omega_{s}}\|\nabla v\|_{\Omega_{s}} \leq \frac{1}{\delta}\left\|\nabla u_{c}\right\|_{\Omega_{s}}^{2}+\delta \epsilon_{s}^{2}\|\nabla v\|_{\Omega_{s}}^{2}
$$

Since $\cosh (t) \geq 1$, it follows that $\ln \left(1-v+v \cosh \left(v+u_{c}\right)\right) \geq 0$ and hence $E(v) \geq$ $C(\epsilon, \delta)\|\nabla v\|^{2}-\frac{1}{\delta}\left\|\nabla u_{c}\right\|_{\Omega_{s}}^{2}$. By choosing $\delta$ sufficiently small, $C(\epsilon, \delta)>0$ is satisfied. Then the Poincaré-Friedrich's inequality gives us

$$
E(v) \geq C(\epsilon, \delta)\|v\|_{1}^{2}+C\left(u_{c}, g\right)
$$

which shows the coercivity of $E$. Finally, Theorem 3.3.4 in [30] shows the existence of the minimizer. Since $E$ is strictly convex, the minimizer is unique. 


\section{Finite Element Discretization}

With the theoretical framework of weak solutions presented in the previous section, we next consider the implications on associated finite element discretizations. In particular, we show ellipticity of the bilinear form, $A(.,$.$) , and establish several properties on the functional$ $N(u)$. From this we are able to verify optimality through an a priori error estimate and demonstrate convergence in the $H^{1}$-norm.

This section illustrates the numerical robustness of the finite element method for the MPBE relative to the unmodified equation. Convergence proofs for solutions of the unmodified PBE include restrictive mesh conditions [15] which are generally not satisfied by standard meshing software designed for the PBE, e.g. GAMer [31]. These conditions arise from the need for the discrete solution to satisfy $L^{\infty}$ estimates. In this section, we show that the MPBE does not have the same restrictions, as the nonlinearity is bounded. As a result, this yields a provably convergent method for the MPBE using meshes generated by existing meshing software, without the need to satisfy the discrete $L^{\infty}$ bounds. We also note that the linear systems arising from discretization of the MPBE are better conditioned (compared to the PBE), which leads to a more efficient numerical method.

\subsection{Analysis of the Nonlinear Finite Element Approximation}

Consider $u_{D}$ that satisfies the Dirichlet boundary conditions in (8). We then solve for $u_{0} \in H_{0}^{1}(\Omega)$ with $u_{0}=u-u_{D}$. Correspondingly, we define $V^{h}$ to be the space of globally continuous piecewise linear elements that satisfy Dirichlet boundary conditions. That is $V^{h}=\left\{v \in H^{1}(\Omega),\left.v\right|_{\tau} \in \mathcal{P}_{1}(\tau), v=g-u_{c}\right.$ on $\left.\partial \Omega, \forall \tau \in \mathcal{T}_{h}\right\}$ where $\mathcal{T}_{h}$ is the triangulation of $\Omega$, and $V_{0}^{h}=V^{h} \cap H_{0}^{1}(\Omega)$. From this, we consider the finite element approximation based on (11) to be: Find $u_{h} \in V^{h}$ such that

$$
A\left(u_{h}, v_{h}\right)+\left(N\left(u_{h}\right), v_{h}\right)+\left\langle f_{c}, v_{h}\right\rangle=0, \quad \forall v_{h} \in V_{0}^{h} .
$$

Several results hold in this discrete setting as a direct consequence of the theory provided in the previous sections. From Lemma 3.1, for example, we have that $u_{h}$ is the minimizer of $E$ in $V^{h}$. Also, since $V^{h}$ is a convex and closed subset of $H^{1}(\Omega)$, based on Theorem 3.2, existence and uniqueness of the discrete solution follows as well.

For finite element approximations it is important to verify not only the existence of a unique solution, but also optimality of the solution. We present this result below in Theorem 4.2 and follow in Theorem 4.3 by establishing convergence of the discrete solution as the mesh is refined. We use several results in functional analysis that are instrumental in proving our proposed theory.

The following lemma establishes important properties of bilinear form $A(.,$.$) and func-$ tional $N($. ), which are then used to show optimality of the discrete solution in Theorem 4.2. We choose to present the statement of the lemma and theorem to coincide with the unmodified Poisson-Boltzmann equation theory as in Lemma 6.1 and Theorem 6.2 in [15]. The relationship between the results of the PBE and the MPBE in this context is important, but it is notable that our hypothesis and assumptions differ from the existing PBE theory. As a result, the mechanics of our proofs are different and the simplicity of our theory further highlights the accessibility and ease-of-use of a modified form of the Poisson-Boltzmann equation. 
Lemma 4.1 (Properties of $A(.,$.$) and N($.$) )$

(a) The bilinear form $A(u, v)$ is coercive and bounded. That is

$$
c\|u\|_{1}^{2} \leq A(u, u), \quad \text { and } \quad A(u, v) \leq C\|u\|_{1}\|v\|_{1} .
$$

(b) The operator $N$ satisfies the property

$$
(N(u)-N(v), u-v) \geq 0 .
$$

(c) The operator $N$ is bounded in the sense that for $u, v \in H^{1}(\Omega), w \in L^{2}(\Omega)$,

$$
(N(u)-N(v), w) \leq K\|u-v\|\|w\| .
$$

Proof The proof of (a) is standard.

To prove (b), consider the functional $R(u)=\int_{\Omega} \bar{\kappa} \ln (1-v+v \cosh (u))$. From the proof of Theorem 3.2, we have that $R$ is a convex and Gâteaux differentiable with $D R(u)[v]=$ $\int_{\Omega} N(u) v=(N(u), v)$. Using the convexity and differentiability of $R$ [32, Theorem 5.3.17],

$$
(D R(u)-D R(v))[u-v]=(N(u)-N(v), u-v) \geq 0 .
$$

To show (c), we use the Cauchy-Schwarz inequality,

$$
(N(u)-N(v), w) \leq\|N(u)-N(v)\|\|w\| .
$$

Now consider $n(t)=\bar{\kappa}_{s}^{2} \frac{\sinh (t)}{1-v+v \cosh (t)}$ as a function from $\mathbb{R}$ to $\mathbb{R}$. Then,

$$
\begin{aligned}
n^{\prime}(t) & =\bar{\kappa}_{s}^{2} \frac{v+(1-v) \cosh (t)}{(1-v+v \cosh (t))^{2}}=\bar{\kappa}_{s}^{2} \frac{v \operatorname{sech}(t)+1-v}{\cosh (t)(\operatorname{sech}(t)-v \operatorname{sech}(t)+v)^{2}} \\
& \leq \frac{\bar{\kappa}_{s}^{2}}{(\operatorname{sech}(t)-v \operatorname{sech}(t)+v)^{2}} \leq \frac{\bar{\kappa}_{s}^{2}}{v^{2}}=K .
\end{aligned}
$$

Then for any $v \in L^{2}(\Omega)$ we have

$$
\begin{aligned}
\|D N(v)\| & =\sup _{w \in L^{2}(\Omega)} \frac{\|D N(v)[w]\|_{L^{2}(\Omega)}}{\|w\|_{L^{2}(\Omega)}} \\
& \leq \sup _{w \in L^{2}(\Omega)} \frac{\sqrt{\int_{\Omega}\left(n^{\prime}(v) w\right)^{2}}}{\|w\|_{L^{2}(\Omega)}} \leq \sup _{w \in L^{2}(\Omega)} K \frac{\|w\|_{L^{2}(\Omega)}}{\|w\|_{L^{2}(\Omega)}}=K .
\end{aligned}
$$

Finally, from the generalized Mean-Value Theorem for Gâteaux differentiable functions [32, Proposition 5.3.11], we have

$$
\|N(u)-N(v)\| \leq K\|u-v\|,
$$

which shows (c).

With these properties on bilinear form $A(.,$.$) and functional N($.$) , one can show that$ the discrete solution is quasi-optimal in the $H^{1}$-norm. This result resembles Céa's lemma, which shows quasi-optimality of discrete solutions for bilinear forms. 
Theorem 4.2 (Quasi-optimal a priori error estimate) Let $u$ and $u_{h}$ be the solution of RMPBE and its finite element approximation, respectively. Then the finite element solution $u_{h}$ is quasi optimal, i.e.

$$
\left\|u-u_{h}\right\|_{1} \leq C \inf _{v_{h} \in V^{h}}\left\|u-v_{h}\right\|_{1} .
$$

Proof The proof directly follows the proof of [15, Theorem 6.2].

From this a priori estimate we are now able to relate the error to a given mesh in the following convergence result.

Theorem 4.3 (Convergence in norm of the discrete solution) Let $u \in H^{1}(\Omega)$ be the weak solution of RMPBE and $u_{h}$ be the finite element approximation. Further we assume the following

- The interface $\Gamma$ is polygonal and is exactly represented by the faces of simplices in $\mathcal{T}_{h}$.

- The maximum diameter of the elements in the mesh, $h$, goes to 0.

- The family of triangulations $\mathcal{T}_{h}$ is shape regular.

Then there exists a constant $C$ independent of $h$ such that

$$
\left\|u-u_{h}\right\|_{1, \Omega} \leq C h\left(\sum_{\tau \in \mathcal{T}_{h}}|u|_{2, \tau}\right)^{1 / 2}
$$

and hence

$$
\lim _{h \rightarrow 0}\left\|u-u_{h}\right\|_{1}=0 .
$$

Proof Because of our assumption on the interface $\Gamma, \epsilon$ and $\bar{\kappa}$ are piecewise constants on each element $\tau \in \mathcal{T}_{h}$. If $\tau \subset \Omega_{m}$, then for $v \in H_{0}^{1}(\tau)$ we extend it by 0 outside $\tau$ to $\tilde{v}$ so that $\tilde{v} \in H_{0}^{1}(\Omega)$. By definition of the weak solution we have,

$$
\begin{aligned}
(\epsilon \nabla u, \nabla \tilde{v})_{\Omega} & =0, \\
\epsilon_{m}(\nabla u, \nabla v)_{\tau} & =0 \quad \forall v \in H_{0}^{1}(\tau) .
\end{aligned}
$$

A regularity result of elliptic partial differential equations [33] says that $u \in H^{2}(\tau)$ for any $\tau \subset \Omega_{m}$. Similarly, since $N(u) \in L^{2}(\Omega)$ we have $u \in H^{2}(\tau)$ for any $\tau \subset \Omega_{s}$. Letting $I_{h} u \in V^{h}$ be the finite element interpolant of $u$, our quasi-optimal result (17) and standard approximation theory [34] results in

$$
\left\|u-u_{h}\right\|_{1}^{2} \leq C\left\|u-I_{h} u\right\|_{1}^{2}=C \sum_{\tau \in \mathcal{T}_{h}}\left\|u-I_{h} u\right\|_{1, \tau}^{2} \leq C h^{2} \sum_{\tau \in \mathcal{T}_{h}}|u|_{2, \tau}^{2},
$$

which completes the proof.

\subsection{Nonlinear Solve}

The previous sections establish important results regarding the weak form of the nonlinear problem. In this section we examine the linearized modified Poisson-Boltzmann equation. 
We begin by defining the functional $F(\cdot)$ by

$$
\begin{aligned}
\langle F(u), v\rangle= & (\epsilon \nabla u, \nabla v)+\left(\bar{\kappa}^{2} \frac{\sinh \left(u+u_{c}\right)}{1-v+v \cosh \left(u+u_{c}\right)}, v\right) \\
& +\left(\left(\epsilon-\epsilon_{m}\right) \nabla u_{c} \cdot \nabla v\right) .
\end{aligned}
$$

Using $F$, the nonlinear weak problem given in (11) can be restated as: find $u \in H^{1}(\Omega)$ such that

$$
\langle F(u), v\rangle=0 \quad \forall v \in H_{0}^{1}(\Omega) .
$$

\subsection{Newton Linearization}

To solve the nonlinear equation in (19), we apply Newton's method, which uses the linearization of the nonlinear form $\langle F(u), v\rangle$. Thus, for the MPBE, the bilinear form $\langle D F(u)[w], v\rangle$ is given by

$$
\langle D F(u)[w], v\rangle=(\epsilon \nabla w, \nabla v)+\left(\bar{\kappa}^{2} \frac{v+(1-v) \cosh \left(u+u_{c}\right)}{\left(v \cosh \left(u+u_{c}\right)-v+1\right)^{2}} w, v\right) .
$$

Newton's method with damping $(\gamma \in[0,1])$ solves for the unknown $u$, which can be summarized by

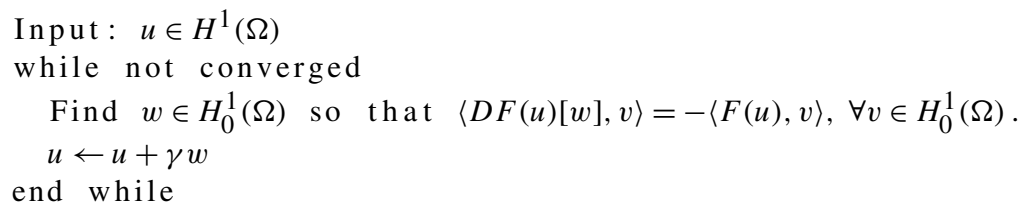

For the discrete problem, the spaces $H^{1}(\Omega)$ and $H_{0}^{1}(\Omega)$ are replaced by $V^{h}$ and $V_{0}^{h}$ respectively. Convergence criteria for a damped Newton's method may be $\left|\left\langle F\left(u_{h}\right), v_{h}\right\rangle\right|<T O L$ for all $v_{h}$ or $\left\|u_{h}^{i+1}-u_{h}^{i}\right\|<T O L$, where $T O L$ is some user specified tolerance and $u_{h}^{i}$ and $u_{h}^{i+1}$ are approximations to the solution from successive iterations of the algorithm. In our experiments we found that convergence of the damped Newton iterates was insensitive to the method of selecting $\gamma$.

The Newton iteration above requires an initial approximation to the solution and convergence is dependent on this selection. Moreover, an accurate initial approximation leads to improved performance. For our approach, we use the solution of a linearized PBE, where the linearized PBE arises from linearizing the nonlinear term in either the PBE or MPBE. For example, near $\tilde{u}=0$, the linearized PBE or MPBE is

$$
-\nabla \cdot(\epsilon(x) \nabla \tilde{u}(x))+\bar{\kappa}^{2}(x) \tilde{u}(x)=4 \pi \beta e_{c} \sum_{i=1}^{N_{m}} Q_{i} \delta\left(x-x_{i}\right)
$$

This equation is then regularized as described for the nonlinear the MPBE and solved using the finite element method. 


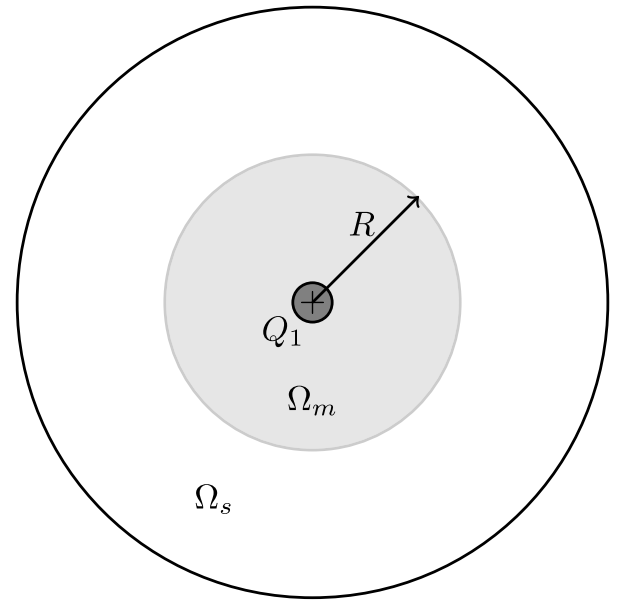

(a) Born Ion

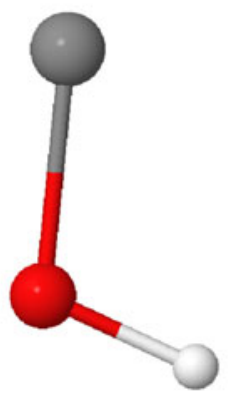

(b) Methanol

Fig. 3 Models used in the numerical experiments

\subsection{Convergence Rate of the Discrete Solution}

Theorem 4.3 states that the expected rate of convergence for discrete solution is $\mathcal{O}(h)$ for the $H^{1}$ norm. For second order elliptic problems with continuous coefficients, one can often prove that the convergence rate for the $L^{2}$ norm is $\mathcal{O}\left(h^{2}\right)$. We are unable to show this rate for the $L^{2}$ norm for the MPBE because the coefficients $\epsilon$ and $\kappa$ are discontinuous. However we show $\mathcal{O}\left(h^{2}\right)$ convergence in $L^{2}$ norm experimentally, and also verify the claim of $\mathcal{O}(h)$ convergence for the $H^{1}$ norm by examining the convergence of our finite-element scheme for the Born ion.

The domain for the Born ion consists of a spherical solute of radius $R$ with a single point charge $Q_{1}$ at its center [35]. The solute is surrounded by a solvent, with the solvent domain truncated at a finite spherical radius, as depicted in Fig. 3a. Analytical solutions for the MPBE do not exist. To obtain a "true" solution, we exploit the spherical symmetry in the Born ion, and recast (8) in spherical coordinates,

$$
-\frac{1}{r^{2}} \frac{d}{d r}\left(r^{2} \epsilon \frac{d u}{d r}\right)+\bar{\kappa}^{2} \frac{\sinh \left(u+u_{c}\right)}{1-v+v \cosh \left(u+u_{c}\right)}=\frac{1}{r^{2}} \frac{d}{d r}\left(r^{2}\left(\epsilon-\epsilon_{m}\right) \frac{d u_{c}}{d r}\right) .
$$

We solve this one-dimensional equation using a finite volume method on a fine mesh, and consider the resulting solution to be the "true" solution.

One advantage of the modified form of the Poisson-Boltzmann equation that we pursue is the natural fit into existing PBE finite element software. We employ the finite element package FETK [36] which is frequently used for PBE computation. The finite element approximation benefits from an accurate meshing of the subdomains and we use a three-dimensional tetrahedral mesh generated using Geometry-preserving Adaptive Mesher (GAMer) [31]. In our convergence results, we normalize the mesh parameter, $h$, by its value for the coarsest mesh, $h_{\max }$. Note that the $L^{2}$ and $H^{1}$ errors are absolute errors, and depend on the size of the domain and choice of units.

As an example, we consider $a=1, \epsilon_{m}=1, \epsilon_{s}=78$, ionic-strength of $0.1 \mathrm{M}$, and temperature of $300 \mathrm{~K}$. Table 1 summarizes the convergence rate of the FEM solution for the MPBE and the results indicate that convergence is approaching optimality. 
Table $1 L^{2}$ convergence of $u$

\begin{tabular}{lclcl}
\hline$h / h_{\max }$ & $L^{2}$ error & $L^{2}$ convergence rate & $H^{1}$ error & $H^{1}$ convergence rate \\
\hline 1 & 247.8 & - & 442.9 & - \\
$1 / 2$ & 75.6 & 1.7 & 201.4 & 1.1 \\
$1 / 4$ & 22.0 & 1.8 & 96.5 & 1.1 \\
$1 / 8$ & 5.7 & 2.0 & 47.1 & 1.0 \\
\hline
\end{tabular}

\subsection{Conditioning of Linear Systems in Newton's Iteration}

The dominant cost in each iteration of Newton's method is the solution of the linear problem, which in this case results in a large, sparse linear system. Consequently we examine bounds on condition numbers from systems arising from unmodified PBE and MPBE to quantify the computational effort needed at each Newton iteration.

For simplicity, initially we consider homogeneous physical parameters $\epsilon=\bar{\kappa}_{s}=1$. Since $\epsilon$ and $\bar{\kappa}$ are common to each equation, the dependence on them in the following discussion of condition numbers is the same.

The condition number of a linear system at a particular Newton iteration depends on the coercivity and continuity constants of the bilinear forms $\langle D F(u)[w], v\rangle$ and $\langle D \tilde{F}(u)[w], v\rangle$, which correspond to the MPBE and PBE respectively. From the Poincaré-Friedrich's inequality and the fact that hyperbolic cosine is a positive function, the coercivity bounds for PBE are

$$
\begin{aligned}
\langle D \tilde{F}(u)[w], w\rangle & =(\nabla w, \nabla w)+\left(\bar{\kappa}^{2} \cosh \left(u+u_{c}\right) w, w\right) \\
& \geq(\nabla w, \nabla w) \geq C\|w\|_{1}^{2} .
\end{aligned}
$$

Furthermore, for boundedness we use the Cauchy-Schwarz inequality,

$$
\begin{aligned}
\langle D \tilde{F}(u)[w], w\rangle & \leq \max _{x \in \Omega_{s}} \cosh \left(u(x)+u_{c}\right)[(\nabla w, \nabla v)+(w, v)] \\
& \leq \max _{x \in \Omega_{s}} \cosh \left(u(x)+u_{c}\right)\|w\|_{1}\|v\|_{1} .
\end{aligned}
$$

For the MPBE, the coercivity bound follows similarly to the PBE case since $\frac{v+(1-v) \cosh (x)}{(v \cosh (x)+1-v)^{2}} \geq 0$. For boundedness, we note that $\frac{v+(1-v) \cosh (x)}{(v \cosh (x)+1-v)^{2}} \leq 1<\frac{1}{v^{2}}$ to get,

$$
\langle D F(u)[w], w\rangle \leq \frac{1}{v^{2}}\|w\|_{1}^{2} .
$$

From these bounds, we argue that the condition number for the PBE could be as large as $\mathcal{O}(\cosh (u))$, while for the MPBE it is $\mathcal{O}\left(1 / v^{2}\right)$. Since $\cosh (u)$ depends exponentially on $u$, the conditioning for the PBE is likely to be considerably worse than for the MPBE if $u$ is large. Table 2 gives the condition numbers (estimated to high accuracy using Lanczos iteration) of the linear systems for the first three iterations of Newton's method for the Born ion, which provides numerical evidence for the above hypothesis. For the MPBE, $v$ corresponding to ionic radius of $a=1$ was used.

Next we examine the $v$-dependence of the condition number of the linear systems arising in the MPBE. Table 3 shows the variation in condition number as the parameter $v$ is changed in the MPBE for the Born ion. Specifically, the table shows the condition numbers 
Table 2 Comparison of condition numbers for the Born ion

Table 3 Condition numbers for varying $v$

Table 4 Condition numbers for varying $\epsilon_{s} / \epsilon_{m}$

Table 5 Condition numbers for varying $\bar{\kappa}_{S}$

\begin{tabular}{lll}
\hline Newton iteration & PBE & MPBE \\
\hline 1 & $3.21 \mathrm{e}+08$ & $1.76 \mathrm{e}+04$ \\
2 & $1.18 \mathrm{e}+08$ & $1.75 \mathrm{e}+04$ \\
3 & $4.34 \mathrm{e}+07$ & $1.75 \mathrm{e}+04$ \\
\hline
\end{tabular}

\begin{tabular}{ll}
\hline$v$ & Condition number \\
\hline $1.2 \mathrm{e}-01$ & $1.79 \mathrm{e}+04$ \\
$1.2 \mathrm{e}-04$ & $1.76 \mathrm{e}+04$ \\
$1.2 \mathrm{e}-07$ & $1.35 \mathrm{e}+06$ \\
$1.2 \mathrm{e}-10$ & $4.34 \mathrm{e}+08$ \\
0 & $3.21 \mathrm{e}+08$ \\
\hline
\end{tabular}

\begin{tabular}{rc}
\hline$\epsilon_{s} / \epsilon_{m}$ & Condition number \\
\hline 1 & $19.2 \mathrm{e}+03$ \\
10 & $9.3 \mathrm{e}+03$ \\
100 & $22.4 \mathrm{e}+03$ \\
1000 & $21.9 \mathrm{e}+04$ \\
10000 & $21.8 \mathrm{e}+05$ \\
\hline
\end{tabular}

\begin{tabular}{cl}
\hline $\bar{\kappa}_{S}$ & Condition number \\
\hline 0 & $1.78 \mathrm{e}+04$ \\
0.1 & $1.73 \mathrm{e}+04$ \\
1 & $1.77 \mathrm{e}+04$ \\
10 & $2.01 \mathrm{e}+04$ \\
100 & $7.56 \mathrm{e}+04$ \\
\hline
\end{tabular}

of matrices for the first iteration of Newton's method. The results indicate that as $v$ goes to 0 , the conditioning of the linear systems in the Newton iteration deteriorates. Since the $\mathcal{O}\left(1 / v^{2}\right)$ bound on the condition number was derived from coercivity and continuity bounds and since the bounds are not tight, there is not a sharp agreement with the condition numbers seen in practice.

The condition number for $v=0$ corresponds to the unmodified PBE, for which we have already seen that the condition number is bounded by $\mathcal{O}(\cosh (u))$. This explains why the condition number does not increase as $\mathcal{O}\left(1 / v^{2}\right)$, but stays bounded as $v$ is decreased.

Finally we examine the dependence of the condition number as the parameters $\epsilon$ and $\bar{\kappa}_{s}$ are varied. Table 4 and 5 show the variation in condition numbers for the first iteration of Newton's method. We observe only a small change in the condition number as $\bar{\kappa}_{s}$ is increased by four orders of magnitude, which is expected since $\bar{\kappa}$ effects the mass matrix part of the full assembled matrix. On the other hand, condition number is more sensitive to the jump $\epsilon_{s} / \epsilon_{m}$ and varies linearly as expected. 


\section{Solution to the Nonlinear PBE using the MPBE}

The modified form of the PBE that we consider in this paper is motivated from physics and results in a tractable model from a theoretical point-of-view. In addition to the advantageous numerical properties, such as optimal accuracy and improved conditioning, the MPBE is also a useful tool when considering the unmodified PBE. Indeed, the finite element approach to the MPBE fits well into an existing finite element computational framework for the PBE. Even more, as we motivate in this section, it is also a valuable approach for generating an initial guess for the unmodified PBE.

As the ion size goes to 0 in the MPBE, we recover the PBE. Yet, for a finite-size with $v>0$, the nonlinearity in the MPBE is numerically evaluated more effectively. To see this, consider the forms $\langle\tilde{F}(u),(v)\rangle$ and its linearization $\langle D \tilde{F}(u)[w], v\rangle$ for the PBE

$$
\begin{aligned}
\langle\tilde{F}(u),(v)\rangle & =(\epsilon \nabla u, \nabla v)+\left(\bar{\kappa}^{2} \sinh \left(u+u_{c}\right), v\right)+\left(\left(\epsilon-\epsilon_{m}\right) \nabla u_{c} \cdot \nabla v\right), \\
\langle D \tilde{F}(u)[w], v\rangle & =(\epsilon \nabla w, \nabla v)+\left(\bar{\kappa}^{2} \cosh \left(u+u_{c}\right) w, v\right)+\left(\left(\epsilon-\epsilon_{m}\right) \nabla u_{c} \cdot \nabla v\right) .
\end{aligned}
$$

In particular, each step of a Newton iteration requires evaluation of $\cosh \left(u+u_{c}\right)$ and $\sinh \left(u+u_{c}\right)$. If the initial approximation is not close to the solution, then these hyperbolic quantities may be large, leading to overflow. One method of avoiding this issue is by capping the input [37]. However, even with truncation the resulting solution process may be complex. We propose using the MPBE as an initial guess in Newton's method to solve the unmodified PBE and we investigate this in the remainder of the paper.

To highlight the differences with the MPBE, we consider the nature of the nonlinearities. The nonlinearities in $\langle F(u), v\rangle$ and $\langle D F(u)[v], w\rangle$ for the MPBE (see (18) and (20)) are $N\left(u+u_{c}\right)$ and $N^{\prime}\left(u+u_{c}\right)$, which are bounded by $\bar{\kappa}_{s}^{2} / v$ and $\bar{\kappa}_{s}^{2} / \nu^{2}$ respectively. Moreover if the input to hyperbolic functions are large, the nonlinearities in MPBE go to 0.

Thus, if a solution to the unmodified PBE is desired-without taking into account steric effects - using the MPBE solution provides a physically accurate and computationally tractable initial approximation. For example, choosing a small ion size so that $1 / v^{2}$ is modest in size may provide a reasonable initial approximation for the PBE. This hypothesis is experimentally verified for the Born ion and for methanol (see Fig. 3). The parameters and subdomains for methanol were obtained from the APBS software package and meshed using GAMer [31].

For a comparison, we consider solving the nonlinear PBE with either a linear approximation of the PBE (labeled PBE in Table 6) or with the nonlinear MPBE (labeled MPBE in Table 6). In the case where the MPBE is used to form an initial approximation to the PBE, we report the sum of the Newton iterations from solving both the PBE and MPBE. An ion size of $10^{-1}$ is used in the MPBE, but other sizes are also effective. In both ion configurations, using the MPBE accelerates convergence of the nonlinear PBE. The improvement is more pronounced for methanol, which is particularly difficult to solve without using the MPBE to generate the initial guess. In the case of the Born ion, the linear approximation provides an adequate initial approximation, which unlike methanol, has a more uniform and basic geometry. Here we see that using the MPBE may provide a significant improvement in the convergence of the Newton iteration.

Remark 5.1 As noted earlier, convergence of Newton's method is highly dependent on choosing a suitable initial approximation. One way to generate this is to use homotopy continuation methods [38]. A homotopy $H(x, t)$ between two functions $f(x)$ and $g(x)$ from a space topological space $X$ to another space $Y$ is a continuous function from $X \times[0,1]$ such 
Table 6 Comparison of Newton iterations for solving the PBE

\begin{tabular}{lcl}
\hline Ion & Initialize with linear PBE & Initialize with MPBE \\
\hline Born & 17 & 14 \\
Methanol & 121 & 21 \\
\hline
\end{tabular}

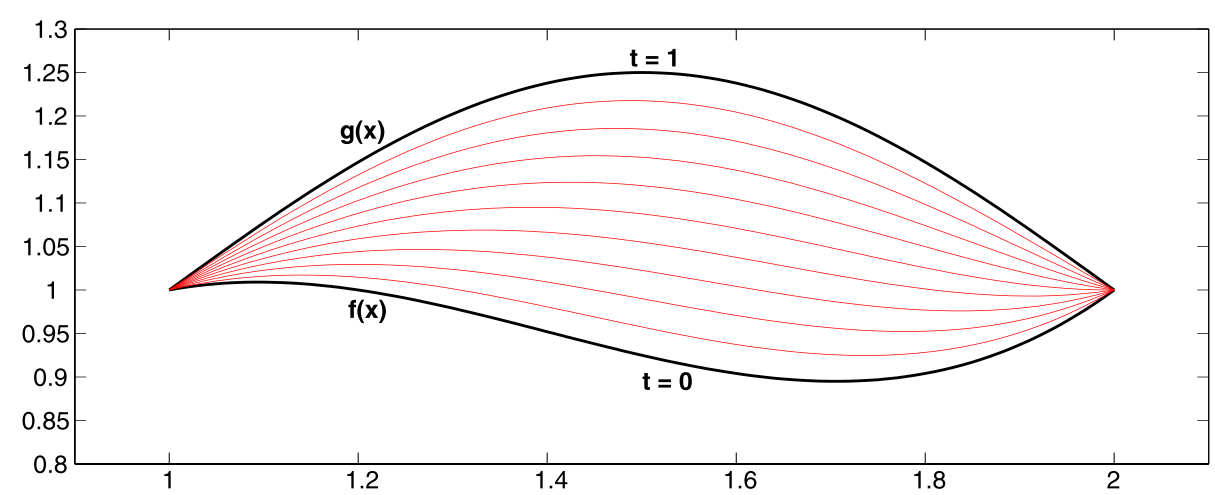

Fig. 4 Homotopy between two functions

$H(x, 0)=f(x)$ and $H(x, 1)=g(x)$ for all $x \in X$, see Fig. 4. If we want to find zeros of $g$ using Newton's method, then in homotopy continuation methods the function $f$ is chosen so that it is easy to solve for $t=0$, and as the parameter $t$ varies from 0 to 1 , the initial guess for $H\left(\cdot, t_{n}\right)$ is generated from the solution of $H\left(\cdot, t_{n-1}\right)$. Homotopy methods aim to accelerate the solution of the target problem $(t=1)$ by solving a sequence of modified problems, as $t$ increases from 0 to 1 .

For solving the PBE, we designate $g(\cdot)$ to be the nonlinear function corresponding to the FEM formulation of PBE in (21). The function $f(\cdot)$ is defined as the nonlinear function corresponding to the MPBE in (18). Varying $t$ from 0 to 1 then corresponds to varying $v$ in MPBE from some small initial value to 0 . This is equivalent to solving MPBE with decreasing ion size in successive nonlinear solves until the ion size is 0 , at which point we solve the PBE. In our numerical experiments we use only one partition of the homotopy map, but for more difficult problems, more homotopy resolution may be necessary.

\section{Conclusion}

In this paper we have considered finite element approximations to a modified form of the Poisson-Boltzmann equation. The modified equation is important as it accounts for the steric or finite-size effects of ion interactions in a molecular simulation. Previously, modeling and computation efforts using this equation have been considered, but in this paper we established several important theoretical results regarding the modified Poisson-Boltzmann equation and related the modified form to the standard form. In particular, we established existence and uniqueness of a weak solution to the problem thereby enabling a finite element formulation. To this end, we also showed convergence and optimal mesh dependence of a finite element approximation to the problem. As a practical consideration we examined the relationship between the conditioning and the standard Poisson-Boltzmann equation, showing that the conditioning is improved for the modified form and that the relationship is 
dependent on the ion size in a consistent manner. We also discussed the numerical evaluation of the modified equation, showing that numerical artifacts of evaluation in the standard form are not present in the modified form. Finally, we discussed the use of the modified equation to assist in the iterative solution of the nonlinear Poisson-Boltzmann equation, ultimately reducing the total number of linear solves and total time to solution.

\section{References}

1. Davis, M.E., McCammon, J.A.: Electrostatics in biomolecular structure and dynamics. Chem. Rev. 90(3), 509-521 (1990)

2. Koehl, P.: Electrostatics calculations: latest methodological advances. Curr. Opin. Struct. Biol. 16(2), 142-151 (2006)

3. Vizcarra, C.L., Mayo, S.L.: Electrostatics in computational protein design. Curr. Opin. Chem. Biol. 9(6), 622-626 (2005)

4. Gouy, G.: Sur la constitution de la charge électrique a la surface d'un électrolyte. J. Phys. Theor. Appl. 9, 455-468 (1910)

5. Chapman, D.L.: A contribution to the theory of electrocapillarity. Philos. Mag. 25, 457-481 (1913)

6. Borukhov, I., Andelman, D., Orland, H.: Steric effects in electrolytes: a modified Poisson-Boltzmann equation. Phys. Rev. Lett. 79(3), 435-438 (1997)

7. Borukhov, I., Andelman, D., Orland, H.: Adsorption of large ions from an electrolyte solution: a modified Poisson-Boltzmann equation. Electrochim. Acta 46(2-3), 221-229 (2000)

8. Khair, A.S., Squires, T.M.: Ion steric effects on electrophoresis of a colloidal particle. J. Fluid Mech. 640, 343-356 (2009)

9. Bikerman, J.J.: Structure and capacity of electrical double layer. Philos. Mag. 33(220), 384-397 (1942)

10. Kilic, M.S., Bazant, M.Z., Ajdari, A.: Steric effects in the dynamics of electrolytes at large applied voltages. I. Double-layer charging. Phys. Rev. E 75(2), 021502 (2007)

11. Storey, B.D., Edwards, L.R., Kilic, M.S., Bazant, M.Z.: Steric effects on ac electro-osmosis in dilute electrolytes. Phys. Rev. E 77(3), 036317 (2008)

12. Levine, S., Bell, G.M.: Theory of a modified Poisson-Boltzmann equation. I. The volume effect of hydrated ions. J. Phys. Chem. 64(9), 1188-1195 (1960)

13. Outhwaite, C.W., Bhuiyan, L., Levine, S.: Theory of the electric double layer using a modified PoissonBoltzmann equation. J. Chem. Soc. Faraday Trans. 2, Mol. Chem. Phys. 76, 1388-1408 (1980)

14. Tang, Z., Scriven, L.E., Davis, H.T.: A three-component model of the electrical double layer. J. Chem. Phys. 97(1), 494-503 (1992)

15. Chen, L., Holst, M.J., Xu, J.: The finite element approximation of the nonlinear Poisson-Boltzmann equation. SIAM J. Numer. Anal. 45(6), 2298-2320 (2007)

16. Orttung, W.H.: Direct solution of the Poisson equation for biomolecules of arbitrary shape, polarizability density, and charge distribution. Ann. N.Y. Acad. Sci. 303, 22-37 (1977)

17. Cortis, C.M., Friesner, R.A.: Numerical solution of the Poisson-Boltzmann equation using tetrahedral finite-element meshes. J. Comput. Chem. 18, 1591-1608 (1997)

18. Holst, M.J., Baker, N.A., Wang, F.: Adaptive multilevel finite element solution of the Poisson-Boltzmann equation I: algorithms and examples. J. Comput. Chem. 21, 1319-1342 (2000)

19. Baker, N.A., Holst, M.J., Wang, F.: Adaptive multilevel finite element solution of the Poisson-Boltzmann equation II: refinement at solvent accessible surfaces in biomolecular systems. J. Comput. Chem. 21, $1343-1352(2000)$

20. Shestakov, A.I., Milovich, J.L., Noy, A.: Solution of the nonlinear Poisson-Boltzmann equation using pseudo-transient continuation and the finite element method. J. Colloid Interface Sci. 247(1), 62-79 (2002)

21. Xie, D., Zhou, S.: A new minimization protocol for solving nonlinear Poisson-Boltzmann mortar finite element equation. BIT 47(4), 853-871 (2007)

22. Wenbin, C., Yifan, S., Qing, X.: A mortar finite element approximation for the linear Poisson-Boltzmann equation. Appl. Math. Comput. 164(1), 11-23 (2005)

23. Bond, S.D., Chaudhry, J.H., Cyr, E.C., Olson, L.N.: A first-order system least-squares finite element method for the Poisson-Boltzmann equation. J. Comput. Chem. 31(8), 1625-1635 (2010)

24. Zhou, Z., Payne, P., Vasquez, M., Kuhn, N., Levitt, M.: Finite-difference solution of the PoissonBoltzmann equation: complete elimination of self-energy. J. Comput. Chem. 17(11), 1344-1351 (1996)

25. Li, B.: Minimization of electrostatic free energy and the Poisson-Boltzmann equation for molecular solvation with implicit solvent. SIAM J. Math. Anal. 40(6), 2536-2566 (2009) 
26. Frenkel, D., Smit, B.: Understanding Molecular Simulation, 2nd edn. Academic Press, New York (2002)

27. Yu, S., Geng, W., Wei, G.W.: Treatment of geometric singularities in implicit solvent models. J. Chem. Phys. 126(24), 244108 (2007)

28. Fogolari, F., Zuccato, P., Esposito, G., Viglino, P.: Biomolecular electrostatics with the linearized Poisson-Boltzmann equation. Biophys. J. 76(1), 1-16 (1999)

29. Kurdila, M.Z.A.: Convex Functional Analysis, Systems \& Control: Foundations \& Applications. Birkhäuser, Basel (2005)

30. Attouch, H., Buttazzo, G., Michaille, G.: Variational analysis in Sobolev and BV Spaces: Applications to PDEs and Optimization. MPS-SIAM Series on Optimization, vol. 6. SIAM, Philadelphia (2006)

31. Yu, Z., Holst, M., Cheng, Y., McCammon, J.A.: Feature-preserving adaptive mesh generation for molecular shape modeling and simulation. J. Mol. Graph. Model. 26(8), 1370-1380 (2008)

32. Atkinson, K.E., Han, W.: Theoretical numerical analysis: a functional analysis framework, 2 nd edn. Springer, Berlin (2005)

33. Gilbarg, D., Trudinger, N.S.: Elliptic Partial Differential Equations of Second Order, 2nd edn. Springer, Berlin (1998)

34. Braess, D.: Finite Elements: Theory, Fast Solvers, and Applications in Elasticity Theory, 3rd edn. Cambridge University Press, Cambridge (2007)

35. Born, M.: Volumen und hydratationswärme der ionen. Z. Phys. 1, 45-48 (1920)

36. Holst, M.: Adaptive numerical treatment of elliptic systems on manifolds. Adv. Comput. Math. 15(1-4), 139-191 (2001)

37. Baker, N.A., Sept, D., Joseph, S., Holst, M.J., McCammon, J.A.: Electrostatics of nanosystems: application to microtubules and the ribosome. Proc. Natl. Acad. Sci. USA 98, 10037-10041 (2001)

38. Allgower, E.L., Georg, K.: Introduction to Numerical Continuation Methods. Classics in Applied Mathematics, Society for Industrial and Applied Mathematics, vol. 45. SIAM, Philadelphia (2003) 